Vol. 72, N. ${ }^{\circ}$ II5 (mayo 2020), 5I-67

\title{
ECUADOR: LA PRUDENCIA FINANCIERA DE LA BANCA PRIVADA EN DOLARIZACIÓN (2001-2019)
}

\section{ECUADOR: THE FINANCIAL PRUDENCE OF PRIVATE BANKING DURING THE DOLLARIZATION (2001-2019)}

\author{
GONZALO J. PAREDES, ${ }^{1}$ NOEMÍ BRENTA, ${ }^{2}$ ÁNGEL MARIDUEÑA L., ${ }^{3}$ MARÍA JOSÉ PAREJA4 \\ 1 Universidad de Guayaquil y Universidad Católica de Santiago de Guayaquil, Ecuador; \\ 2 Universidad de Buenos Aires, Argentina; 3 Universidad Estatal de Milagro, Ecuador; \\ 4 Universidad de Guayaquil, Ecuador
}

\begin{abstract}
Recepción del manuscrito: 24 de abril de 2020
\end{abstract}
Aceptación versión final: 19 de mayo de 2020

\begin{abstract}
RESUMEN El objetivo es evaluar el nivel de prudencia financiera de la banca privada durante la dolarización. El enfoque de la metodología aplicada es cuantitativo, un análisis descriptivo de cuatro ejes: nivel de liquidez, solvencia, el crédito y su riesgo. Se concluye que la banca es profundamente conservadora con base en el histórico de los fondos disponibles, el alto gasto de provisión para créditos incobrables, y la elevada preferencia por la liquidez expresada en el cortoplacismo de los préstamos. Se afirma que este comportamiento ha obstaculizado un crecimiento alto y sostenido porque contribuye a la ineficiencia de la economía ecuatoriana.
\end{abstract}

PALABRAS ClAVE Banca privada, riesgo, crédito, dolarización, Ecuador.

ABSTRACT The objective is to evaluate financial prudence of private banking during the dollarization. The focus of the applied methodology is quantitative, a descriptive analysis of four axes: level of liquidity, solvency, credit and its risk. It is concluded that banks are deeply conservative based on the history of available funds, the high expending on provision for bad loans and the high preference for liquidity expressed in the short-termism of loans. It is stated that this behaviour has constrained high and sustained growth since it leads to inefficiency of the Ecuadorian economy.

KEYWORDS Private banking, risk, credit, dollarization, Ecuador.

JEL CODES E12, G21, N26, O16.

\section{INTRODUCCIÓN}

La banca privada cumple con el rol de trasladar liquidez a los inversionistas y, a la vez, precautelar la devolución de los ahorros a los depositantes. La sola presunción de crisis económica 
provocaría que prioricen esta última en desmedro de la primera. Por lo tanto, las entidades financieras enfrentan diferentes tipos de riesgos, y también incertidumbre. Los primeros se pueden medir, pero la segunda no. No obstante, mientras mayores sean los riesgos, la economía está cada vez más expuesta a la crisis si se cumplen los eventos inciertos.

Para afrontar los riesgos y atenuar la incertidumbre, la banca privada conforma fondos de liquidez, fortalece su patrimonio, realiza provisiones para probables pérdidas, y reduce los plazos y montos de las líneas de crédito a conceder. Sin embargo, las instituciones financieras pueden no cumplir con estas medidas o exacerbarlas. En el primer caso, provocan el debilitamiento del patrimonio hasta una crisis sistémica, mientras que en el segundo se acumulan recursos en exceso con repercusiones en la sostenibilidad del crecimiento al no contar con crédito productivo suficiente. De esta manera, la interrogante que surge en un país dolarizado como Ecuador es ¿qué tan prudente o conservadora es la banca privada para enfrentar los diferentes tipos de riesgos y la incertidumbre?

Para llevar a cabo este estudio, el enfoque de la metodología aplicada es cuantitativa. Se analiza la orientación del crédito bancario, los indicadores de liquidez y solvencia, y el riesgo expresado a través del nivel de provisión para pérdidas crediticias, la morosidad y la cartera vencida. Esta investigación se impulsó porque se considera que en los sistemas financieros de los países en desarrollo la banca privada tiene un peso importante y su desenvolvimiento influye en el proceso de acumulación del capital, el crecimiento de la producción, la capacidad exportable y el desarrollo económico.

Por lo tanto, dado el peso de la banca privada en el sistema financiero ecuatoriano, las necesidades de financiamiento de los sectores productivos, el nivel de rentabilidad con respecto a otros sectores económicos, la amplia brecha de productividad con los países de la región y con otras economías de tamaño similar, el déficit del comercio exterior y una economía sin moneda propia, son elementos y características de la relación entre las finanzas con el sector real de Ecuador que lo convierten en un caso de análisis relevante para la academia y las políticas públicas.

El artículo se organiza en cinco secciones. En la primera, se bosquejan los elementos teóricos y conceptuales necesarios para abordar la pregunta de investigación propuesta; en la segunda, se realiza una breve descripción sobre la posición de la banca privada en la economía ecuatoriana y en América Latina; en la siguiente sección, se lleva a cabo el relevamiento empírico con base en los cuatro ejes señalados y en la estadística de la Superintendencia de Bancos, esencialmente; en la cuarta, se explican los resultados encontrados; en la quinta y última parte, se efectúan unas reflexiones finales.

\section{MARCO TEÓRICO}

En los sistemas financieros de los países en desarrollo la banca privada tiene un peso importante y su desenvolvimiento influye en el proceso de acumulación del capital, el crecimiento de la producción, la capacidad exportable y el desarrollo económico. De la Cruz y Alcántara (2011) identifican que los sistemas financieros resuelven tres problemas fundamentales: la aglomeración, distribución y monitoreo del capital, para que este último sea utilizado eficientemente. De acuerdo a esto, Fitzgerald (2007) señala tres características básicas que capturan 
el impacto del desarrollo financiero - o el qué hacer del sistema financiero- sobre el crecimiento económico: (1) nivel de intermediación financiera; (2) la eficiencia de dicha intermediación; y (3) su composición.

Por lo tanto, King y Levine (1993) destacan que los sistemas financieros más avanzados, o más saludables, mejoran la productividad de una economía a través de: (1) la evaluación de los posibles empresarios y de los mejores proyectos de inversión, (2) la movilización de los recursos para financiar proyectos, (3) la diversificación de los riesgos de las actividades innovadoras, y (4) la compensación por participar de la innovación.

No obstante, la actividad bancaria es la creación de dinero a través del crédito, una operación basada en la partida doble. La falta de liquidez y los problemas asociados a la incapacidad de recuperación de los créditos concedidos son riesgos inherentes al funcionamiento de la banca privada. El primero constituye el riesgo de liquidez y está relacionado al «nivel suficiente de activos líquidos de alta calidad que puedan ser transformados en efectivo a efectos de enfrentar necesidades extraordinarias de liquidez en un cierto horizonte de tiempo» (Superintendencia de Bancos, 2019, pág. 6). El segundo se identifica como riesgo de crédito y está fomentado por la asimetría de información. Por lo tanto, se lo calcula con base en la probabilidad de incumplimiento, la exposición al incumplimiento y la severidad de la pérdida.

La importancia de medir y evaluar estos riesgos radica en que los problemas de una institución financiera pueden llegar a ser sistémicos, donde éstos pueden propagarse con mayor rapidez y fuerza mientras más grande sea esta institución. McMillan (2018) afirma que «[...] los pánicos financieros son el talón de Aquiles de la banca [...] coartan la capacidad del sistema financiero de coordinar las actividades económicas, causan enormes distorsiones en los precios y desembocan en grandes recesiones en la economía real» (pág. 22).

Sin embargo, la eficiencia en la intermediación de la banca privada está en colocar los excedentes de liquidez de la economía en proyectos o sectores con alta productividad y de bajo riesgo para luego maximizar la rentabilidad. McMillan (2018) conceptualiza la eficiencia como «la transformación de los riesgos, en general, diversificando y estructurando sus balances» (pág. 43). No obstante, en la búsqueda de ese equilibrio albergan disyuntivas y a menudo las entidades optan por maximizar la rentabilidad en desmedro de financiar proyectos con alta productividad u obtener bajos riesgo de liquidez y crediticio (ver Figura 1).

La trilogía imposible de la figura 1 tiene como base teórica a la corriente poskeynesiana, a través de la reelaboración de la teoría de la preferencia por la liquidez, al enfoque cepalino de la financierización y al racionamiento del crédito de los países en desarrollo. La primera sostiene que el comportamiento bancario está basado en una elección de carteras y no de activos (Cardim de Carvalho, 2015) y que «la preferencia por la liquidez es la principal fuente de ineficiencia macroeconómica en la asignación de recursos» (Hermann, 2014, pág. 74). Keynes (1937) destaca que el ahorro es un fondo rotatorio para satisfacer suministro de crédito o «financiación» requerida para la inversión ex ante, y que:

El Estado tendrá que ejercer una influencia orientadora [...] una socialización bastante completa de las inversiones será el único medio de aproximarse a la ocupación plena; aunque esto no necesita excluir cualquier forma, transacción o medio por los cuales la autoridad pública coopere con la iniciativa privada. (Keynes, 1936, pág. 311) 
Por su parte, el enfoque cepalino sostiene que el sector financiero está cada vez más desconectado del sector real de la economía, aunque el tipo de separación depende mucho si se trata de un país en desarrollo o avanzado. Chena, Bucella y Bosnic (2018) identifican este fenómeno como la «supremacía de las finanzas».

Por último, para los países en desarrollo, la represión financiera puede impulsar la acumulación del capital si los créditos restringidos son los de consumo. Hung (2005) afirma que «si bien una reducción del racionamiento crediticio de los préstamos de consumo impide la acumulación de capital, esta reducción en los préstamos de inversión la facilita» (pág. 322). Para esta argumentación, Hung (2005) conceptualiza a la «trampa del desarrollo» como la mala orientación del crédito bancario, así como la ineficiente intermediación financiera.

La imposibilidad de que la banca privada en un país en vías de desarrollo pueda perseguir los tres objetivos a la vez, se debe a la mala intermediación financiera y al bajo ahorro interno. Por lo tanto, si las entidades financieras no estimulan el ahorro en la población, a pesar del aumento del ingreso per cápita, y cambiar la temporalidad de los depósitos, se ven persistentemente abocadas a racionar el crédito a los sectores con mayor productividad y gestionar el riesgo a través del cortoplacismo, las fuertes garantías, y/o las tasas de interés más altas que permitan mayores provisiones. El bajo desarrollo financiero de estos países se conjuga con la naturaleza de orden privado de los bancos, lo que justificaría la intervención del Estado.

LA LITERATURA SOBRE LOS EFECTOS DEL RIESGO DE CRÉDITO SOBRE LA RENTABILIDAD

Xu et al., (2019) identifican una relación inversa entre la rentabilidad bancaria y su contribución a la toma de riesgo (ver tabla 1 del anexo digital) $;{ }^{2}$ cada vez que la rentabilidad aumenta, las entidades financieras están menos dispuestas a tomar mayores riesgos. Saona (2016), en cambio, mide la relación entre el riesgo de crédito y la rentabilidad y expresa que es directa. En cambio, para Petria et al. (2015) la relación es inversa y estadísticamente significativa.

No obstante, Rodríguez (2015) demuestra para México que la correlación no es significativa si se cambia el tipo de indicador del riesgo de crédito, con base en el debate si es ex post o ex ante. Este autor considera que es mejor usar la proporción de cartera vencida respecto a la cartera total de un banco (la identifica como morosidad) porque este indicador de riesgo de crédito con carácter ex post es «consecuencia de factores económicos, políticos y regulatorios, y varía entre bancos debido a las diferencias en sus políticas de gestión de crédito y de la tecnología y recursos con que operan» (pág. 74).

Para el caso de Ecuador, Vera et al. (2019) determina la inexistencia de la relación entre el riesgo de crédito y la rentabilidad de los bancos. Los autores toman como variable del riesgo de crédito a la provisión por pérdida de préstamos sobre el total de préstamos, y señalan que «este resultado puede sugerir que los bancos ecuatorianos están manteniendo niveles bajos de provisión de préstamos. Esto también podría ser una señal de que el sistema bancario se está relajando» (Vera et al., 2019, pág. 13). 
Figura 1. La trilogía imposible de la banca privada

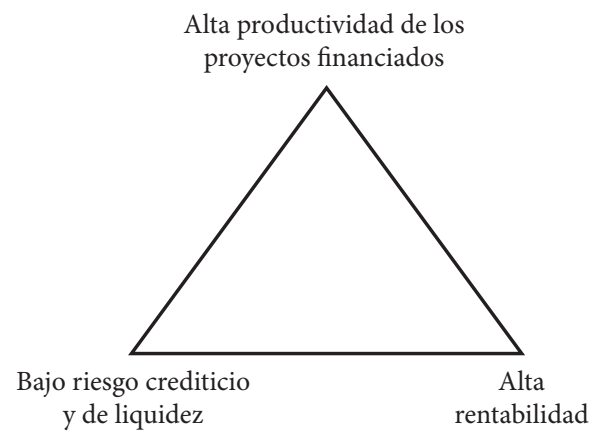

Elaboración: propia.

Vera y Titelman (2013) señalan que el crédito otorgado por la banca privada en América Latina, medido en relación al PIB, es bajo en comparación con las otras regiones del mundo. Además, no ha crecido desde los años noventa. Japón, Estados Unidos, Reino Unido y la eurozona superan en mucho a los países latinoamericanos. Según este estudio, entre los períodos 19901995 y 2006-2011, todas las regiones del mundo aumentaron su profundidad financiera, excepto América Latina que se mantuvo, y Oriente Medio y África del Norte que disminuyeron la participación del crédito bancario en el PIB.

La profundidad financiera se puede medir de dos formas: la suma de los depósitos o de los créditos con respecto al PIB. Si se la mide a través del crédito, Argentina es el país con el indicador más bajo, junto a Ecuador, República Dominicana y Uruguay con respecto a los demás países de América Latina (16 seleccionados) a junio de 2019. No obstante, cuando la medición es por medio de los depósitos, México aparece con la menor profundidad financiera junto a Ecuador, Argentina, República Dominicana, y Nicaragua. Bajo este cálculo, Uruguay ostenta $56,49 \%$ (ver figura 1 del anexo digital).

La profundidad financiera puede diferir al interior de un país. Por ejemplo, México ostenta un indicador con base en los depósitos más bajo que con los créditos. El mismo caso es para Chile y Brasil. Mientras que Uruguay, Guatemala y Argentina tienen una profundidad medida por los depósitos mayor que con los créditos.

Hasta 2016, Ecuador pertenecía a este último grupo, pero la banca privada acortó la diferencia entre los dos tipos de mediciones mediante la aceleración de la tasa de crecimiento de los créditos. Así lo demuestran el cociente entre los depósitos y los créditos: al inicio de la dolarización por cada dos dólares de depósito había uno de crédito. En marzo de 2020 este indicador había disminuido a 1,20 (ver figura 2 del anexo digital).

Es posible reconocer siete etapas de la banca privada (ver Tabla 1), según una categorización realizada con base en su desenvolvimiento, nivel de rentabilidad, los ciclos de la economía, y el peso del Estado a través de la regulación bancaria y el gasto público. La reducción del cociente entre los depósitos y los créditos se evidencia cuando en la última etapa del periodo de estudio (2017-2019) la brecha entre la tasa de crecimiento de los créditos y de los depósitos se 
amplió. Esto demuestra que en el último trienio la banca asumió un mayor riesgo, fue menos conservadora y se encaminó a reducir mucho más el cociente entre depósitos y créditos.

Sin embargo, en esta última etapa las tasas de crecimiento no superaron a sus respectivos promedios, un comportamiento idéntico a las dos anteriores. Los bancos privados para impulsar el crédito a una tasa muy por encima de los depósitos recurrieron al financiamiento externo en 2019 (Tapia, 2020), una decisión pocas veces registrada. Además, fue en la etapa de la primera y segunda «gran expansión» que lograron sobrepasar el promedio de todo el periodo (ver figura 3 del anexo digital).

El efecto de la relajación de los créditos concedidos en relación a los depósitos existentes llevó a la banca a mantener una menor liquidez — fondos disponibles/total de depósitos a corto plazo-. Entre enero de 2017 y diciembre de 2019, la liquidez pasó de 31,1 \% a 25,98 \%. Los más altos indicadores de liquidez se registraron en el primer semestre de 2008 y en el segundo semestre de 2016. A pesar de la disminución de la liquidez de los bancos, el indicador de solvencia - patrimonio técnico constituido/activos y contingentes ponderados por riesgo- ha estado en niveles altos, cerca de los registrados en los años de recesión (2008-2009 y 2016) (ver figura 4 del anexo digital).

Además, la banca privada registró niveles de solvencia muy por encima de lo que sugieren las normas de Basilea III (8\%) y de lo que exige el Código Orgánico Monetario y Financiero (9\%). No obstante, está por debajo de países vecinos como Colombia y Perú, de los dolarizados Panamá y El Salvador, y de las economías más grandes Brasil y México (Asociación de Bancos Privados [AsOBANCA], 2019, pág. 34).

\section{RELEVAMIENTO EMPÍRICO}

\section{LA ORIENTACIÓN DEL CRÉDITO}

El sistema bancario ecuatoriano tenía 24 bancos a diciembre de 2019 (4 con categoría de grande) y se caracteriza por una orientación crediticia de muy corto plazo, altamente líquida, fácilmente reembolsable y dirigida en una mayor cuantía hacia el consumo. Los préstamos de largo plazo están altamente garantizados y, en el periodo estudiado, se destinaron cada vez más hacia el consumo y no a la inversión.

De este modo, la cartera consumo pasó de $29,53 \%$ a 38,34 \% entre 2002 y 2019 (ver Tabla 2), mientras que la cartera comercial disminuyó a 35,36 \% en 2019 cuando ostentaba 60,33\% en 2002. El cambio de la orientación crediticia desde 2002 se comprueba inclusive cuando se suma la cartera de crédito productivo (considerada de largo plazo en más del 80\%) a la comercial: disminuyó de $60,33 \%$ a $45,78 \%{ }^{3}$

No obstante, este cambio ha tenido intermitencias causadas por los shocks externos convertidos en crisis o por el cambio de la política económica. Por ejemplo, en 2015 el gobierno dispuso aumentar el arancel promedio, cambió los precios relativos y restringió fuertemente las importaciones, entre ellas, las de consumo duradero. En consecuencia, la banca privada reorientó el crédito menos hacia el consumo y más hacia el comercial y la construcción. A pesar de este cambio, entre 2002 y 2019, el crédito de largo plazo aumentó su peso en el total, mientras que el de corto plazo disminuyó (ver figura 5 del anexo digital). Esto se explica principalmente 
Tabla 1. Etapas de la banca privada del Ecuador

\begin{tabular}{|c|c|c|c|}
\hline $\begin{array}{l}\text { Etapas de la evolución de la banca privada } \\
\text { ecuatoriana (2000-2015) }\end{array}$ & $\begin{array}{l}\text { Tasa de } \\
\text { crecimiento de } \\
\text { los depósitos }\end{array}$ & $\begin{array}{l}\text { Tasa de } \\
\text { crecimiento } \\
\text { de los créditos }\end{array}$ & Fase del ciclo económico \\
\hline La reconstrucción (2000-2003) & $0,96 \%$ & $1,24 \%$ & Recuperación \\
\hline $\begin{array}{l}\text { La consolidación y la primera gran expansión } \\
(2004-2006)\end{array}$ & $1,55 \%$ & $2,12 \%$ & $\begin{array}{l}\text { Auge (2003-2004) y leve } \\
\text { desaceleración (2005-2006) }\end{array}$ \\
\hline $\begin{array}{l}\text { Los primeros cambios regulatorios } \\
(2007-2009)\end{array}$ & $1,24 \%$ & $0,86 \%$ & Recesión (2008-2009) \\
\hline $\begin{array}{l}\text { La segunda gran expansión: el boom de la } \\
\text { liquidez pública con recursos genuinos (2010- } \\
\text { 2012) }\end{array}$ & $1,29 \%$ & $1,48 \%$ & Auge (2010-2011) \\
\hline $\begin{array}{l}\text { Fin del régimen monetario del neoliberalismo } \\
\text { y el boom de la liquidez pública con recursos } \\
\text { ajenos (2013-2014) }\end{array}$ & $0,86 \%$ & $0,93 \%$ & Leve desaceleración (2014) \\
\hline $\begin{array}{l}\text { La resistencia: los shocks externos y alta } \\
\text { incertidumbre (2015-2016) }\end{array}$ & $0,16 \%$ & $0,14 \%$ & $\begin{array}{l}\text { De la fuerte desaceleración } \\
\text { (2015) a la recesión (2016) }\end{array}$ \\
\hline La tercera gran expansión (2017-2019) & $0,45 \%$ & $1,10 \%$ & $\begin{array}{l}\text { Recuperación (2017) y fuerte } \\
\text { desaceleración (2018-2019) }\end{array}$ \\
\hline
\end{tabular}

Elaboración: propia — con base en Paredes (2015 y 2017) y datos de la Superintendencia de Bancos (2020)—.

Tabla 2. La orientación crediticia por tipo de cartera* $(\%)$

\begin{tabular}{|c|c|c|c|c|c|c|}
\hline Año & $\begin{array}{l}\text { Cartera } \\
\text { comercial }\end{array}$ & $\begin{array}{l}\text { Cartera } \\
\text { consumo }\end{array}$ & $\begin{array}{l}\text { Cartera } \\
\text { vivienda }\end{array}$ & $\begin{array}{l}\text { Cartera } \\
\text { microempresa }\end{array}$ & $\begin{array}{l}\text { Cartera } \\
\text { educativo }\end{array}$ & $\begin{array}{l}\text { Cartera } \\
\text { productivo }\end{array}$ \\
\hline \multicolumn{7}{|l|}{2001} \\
\hline 2002 & $60,33 \%$ & $29,53 \%$ & $8,21 \%$ & $1,93 \%$ & n.d. & n.d. \\
\hline 2003 & $62,51 \%$ & $25,21 \%$ & $9,12 \%$ & $3,16 \%$ & n.d. & n.d. \\
\hline 2004 & $56,93 \%$ & $26,97 \%$ & $11,21 \%$ & $4,88 \%$ & n.d. & n.d. \\
\hline 2005 & $53,55 \%$ & $28,38 \%$ & $11,71 \%$ & $6,36 \%$ & n.d. & n.d. \\
\hline 2006 & $52,05 \%$ & $29,05 \%$ & $11,84 \%$ & $7,06 \%$ & n.d. & n.d. \\
\hline 2007 & $48,60 \%$ & $29,63 \%$ & $13,80 \%$ & $7,97 \%$ & n.d. & n.d. \\
\hline 2008 & $48,91 \%$ & $28,86 \%$ & $13,74 \%$ & $8,49 \%$ & n.d. & n.d. \\
\hline 2009 & $47,13 \%$ & $29,95 \%$ & $14,05 \%$ & $8,87 \%$ & n.d. & n.d. \\
\hline 2010 & $45,89 \%$ & $32,36 \%$ & $13,01 \%$ & $8,75 \%$ & n.d. & n.d. \\
\hline 2011 & $45,23 \%$ & $36,20 \%$ & $9,74 \%$ & $8,83 \%$ & n.d. & n.d. \\
\hline 2012 & $47,83 \%$ & $35,20 \%$ & $8,92 \%$ & $8,03 \%$ & $0,03 \%$ & n.d. \\
\hline 2013 & $49,60 \%$ & $34,67 \%$ & $8,43 \%$ & $7,28 \%$ & $0,02 \%$ & n.d. \\
\hline 2014 & $50,08 \%$ & $34,27 \%$ & $8,25 \%$ & $7,16 \%$ & $0,23 \%$ & n.d. \\
\hline 2015 & $47,13 \%$ & $32,58 \%$ & $9,77 \%$ & $7,66 \%$ & $2,24 \%$ & $0,62 \%$ \\
\hline 2016 & $47,17 \%$ & $30,29 \%$ & $9,78 \%$ & $7,06 \%$ & $2,08 \%$ & $3,62 \%$ \\
\hline 2017 & $41,89 \%$ & $34,16 \%$ & $8,99 \%$ & $6,32 \%$ & $1,73 \%$ & $6,91 \%$ \\
\hline 2018 & $39,12 \%$ & $35,78 \%$ & $8,46 \%$ & $6,19 \%$ & $1,50 \%$ & $8,95 \%$ \\
\hline 2019 & $35,36 \%$ & $38,34 \%$ & $7,92 \%$ & $6,56 \%$ & $1,39 \%$ & $10,42 \%$ \\
\hline
\end{tabular}

*No existen datos disponibles para la cartera de crédito educativo antes de 2012 y para la cartera de crédito productivo antes de 2015 porque se las consideraba parte de otra cartera hasta que las instancias de control dispusieron su creación y aparición en la estadística de la Superintendencia de Bancos.Elaboración: propia — con base en datos de la Superintendencia de Bancos (2020)—. 
mediante el crecimiento de los créditos de consumo de largo plazo en el último trienio: en 2019 aumentó 31,54 \% frente al comercial (se incluyó el productivo) que solo se expandió en 1,34 \%.

La banca privada ecuatoriana es bastante prudente en varios aspectos de la orientación del crédito: (1) con base en la trilogía imposible, el desenvolvimiento de la banca privada ecuatoriana se encuentra en la base del triángulo (ver Figura 1) y le permite maximizar la rentabilidad con bajo riesgo a pesar de orientar el crédito cada vez más hacia el consumo - la cartera con la más alta morosidad-, (2) con otro tipo de combinación estaría renunciando a una elevada rentabilidad o al bajo riesgo crediticio (y de liquidez), (3) la alta preferencia por la liquidez le permite reaccionar rápidamente a shocks externos o al cumplimiento de eventos inciertos, y (4) la cartera de consumo e hipotecaria (con altas garantías) absorbe la mitad del crédito total de largo plazo.

Con respecto a esto último, Paredes y López (2019) señalan que el 55 \% de las empresas del sector manufacturero no se financian con crédito bancario, por lo que están racionadas completamente: más del $61 \%$ de las microempresas y pequeñas empresas son racionadas por el sistema bancario. No obstante, cuando se trata de empresas medianas y grandes, el racionamiento se reduce en menos de la mitad y a 21,43 \%, respectivamente. Asimismo, el restante de este sector $(45 \%)$ ha sufrido algún tipo de racionamiento: por monto, plazo o tasa de interés.

LIQUIDEZ

Desde 2017, el coeficiente de liquidez — fondos disponibles/depósitos a corto plazo- disminuyó a diciembre de 2019 y se ubicó en 25,98 \%. No es el nivel más bajo, pero se acerca a los del primer semestre de 2015 y el tercer trimestre de 2018. Los resultados durante 2019 se deben principalmente a que los créditos crecieron más que los depósitos, y estos últimos aumentaron a una tasa muy baja. En la etapa «resistencia» y «la tercera gran expansión» los depósitos aumentaron en $0,16 \%$ y o,45 \%, respectivamente (ver Tabla 1). El efecto se evidencia con la caída de las reservas realizadas en el Banco Central del Ecuador (вСE) y en bancos e instituciones financieras del exterior. El dinero en caja también disminuyó en 2019 (ver figura 6 del anexo digital).

En junio de 2009, el Gobierno central impuso a la banca privada, a través del Banco Central, la repatriación de capitales por USD 1200 millones (ahorro de los depositantes) para promover el crédito productivo y el consumo. La disposición implicaba que los bancos debían tener por lo menos el 45 \% de sus activos en el país para fortalecer la dolarización, el gobierno insistió con esta medida en los años posteriores. Sin embargo, en noviembre de 2016 la Junta Monetaria y Financiera (formada en el Código Monetario y financiero de 2014) elevó el encaje (depósitos de la banca en el BCE) del 2 al 5\% y excluyó del coeficiente de liquidez doméstica el fondo de liquidez que se invierte en el exterior (ver figura 6 del anexo digital).

Aunque estas medidas impulsaron el crédito interno y movilizaron el ahorro, lo que permitió salir de la crisis en algunos casos y en otros sostener el crecimiento de la economía, la banca redujo su nivel de liquidez en el rubro «bancos e instituciones financieras del exterior», y aumentó débilmente el rubro «bancos e instituciones financieras locales». Por último, con el relajamiento de las regulaciones bancarias a partir de 2017, los fondos depositados en el BCE disminuyeron. 
RIESGO DE CRÉDITO EX ANTE Y EX POST: LAS DOS CARAS DE UNA MISMA MONEDA

Se precisó en el marco teórico que el incumplimiento del prestatario se mide a través del riesgo de crédito. De esta manera, los trabajos de Vera et al. (2019), Xu et al. (2019), Saona (2016) y Petria et al. (2015) establecieron como riesgo de crédito a la provisión de las carteras de créditos. Para evaluar qué tan prudente o conservadora es la banca privada en Ecuador, primero se medirá el riesgo de crédito bajo un enfoque ex ante, a través de las provisiones generadas. En efecto, Saona (2016) señala que los bancos cobran una tasa de interés más alta, si las provisiones por riesgo de incumplimiento son mayores.

El nivel de las provisiones en la banca depende si se miden como un stock o un flujo. En el primer caso, este dato se obtiene del Balance General (ВG) у sirve para calcular los indicadores de cobertura. ${ }^{4}$ El segundo procede del Estado de Pérdidas y Ganancias (EPYG). Con este antecedente, en 2019, el sistema bancario ecuatoriano registró USD 1851,02 millones de provisión acumulada total y USD 503,25 millones en gasto de provisión (ver figura 7 del anexo digital).

Hasta 2012, la provisión acumulada (del balance general) se calculó con base en la pérdida probable de cada uno de los tipos de cartera, de la cartera refinanciada, la reestructurada y de la provisión general de las carteras de crédito. Del 2013 en adelante, se da por la provisión de cada una de las carteras y cuatro nuevos tipos de rubros: (1) provisión genérica por tecnología crediticia, (2) anticíclica, (3) no reversadas, y (4) voluntaria. La «provisión general» desaparece del cálculo. Por lo tanto, para el análisis se consideraron dos tipos de provisión acumulada: (1) la provisión acumulada (solo) de las carteras de crédito, y (2) la provisión acumulada total, donde se considera los «otros rubros». En el EPyG, el gasto que sustenta y contribuye a la acumulación en sus distintos rubros se genera solo con base en las distintas carteras de créditos (Superintendencia de Bancos, 2020).

El comportamiento de las provisiones se relaciona con las etapas del sistema bancario señalado en la tabla 1. En la reconstrucción (2001-2003) y hasta 2004, el primer año de la consolidación, la provisión acumulada total y como gasto disminuyeron. Esto significa que el sistema bancario se depuró de la crisis de 1999 a través de la eliminación de gran parte de las carteras vencidas hasta ese último año. A partir de 2005, la consolidación fue una realidad y a medida que el gasto de provisión se generó, la provisión acumulada total creció, sin excepción, en cualesquiera de esos años (ver figura 8 del anexo digital).

En 2007, 2009, 2010, 2013 y 2018 el gasto de provisión decreció con respecto al año anterior, pero la provisión acumulada total aumentó. En los otros años, el gasto creció más que la acumulada. Esto se puede explicar si se considera en qué cuantía el gasto de provisión cubrió la cartera incobrable y cuánto aportó a la provisión acumulada — como primer paso la que se registró por la suma de las carteras de crédito y, después por la totalidad donde se incluyeron los «otros rubros»-.

Entre 2005 y 2007, más del 80 \% del gasto de provisión se destinó a cubrir la cartera vencida. En 2008, ese porcentaje bajó a 56,12\% y volvió a subir en el trienio posterior, en promedio, a 73 \%. En 2012, disminuyó a 62,57\% y aumentó drásticamente en 2013 a 89,23 \%. En 2014, volvió a descender: 49,54 \%. En 2015 y 2016, lapso de la recesión, fue 82,85 \% y 116,92 \%, respectivamente. ${ }^{5}$ Sin embargo, en 2017 se registró como el gasto que menos cubrió esas pérdidas (41,25\%). En 2018-2019 creció por encima del 80 \% (ver figura 9a del anexo digital). 
Sin embargo, el resultado puede cambiar si se suman los «otros rubros» a la provisión acumulada por las carteras de crédito (ver figura 9b del anexo digital). En todos los años (20052019), excepto 2010, 2015, y 2018, el porcentaje de los gastos de provisión que contribuyó a la cartera vencida fue menor, sobre todo en 2005, 2009, 2011, 2012 y 2017 (el más bajo). Esto significa que el rubro «provisión general» hasta 2012 y la provisión genérica por tecnología crediticia, anticíclica, no reversadas y voluntaria, a partir de 2013 , contribuyeron a incrementar cada año en un mayor importe a la provisión acumulada total (ver figura 9 del anexo digital).

A través del análisis del gasto de provisión por tipo de cartera del EPyG, el crédito de consumo ostenta el $71,76 \%$, mientras que el crédito comercial el 10,78 \% (ver figura 10 del anexo digital). Según la tabla 2, la cartera de crédito de consumo por vencer tuvo un peso sobre el total de carteras de $38,34 \%$ y la comercial de $35,36 \%$. Esto demuestra lo poco riesgosa que se pondera a esta última con respecto a la primera. Asimismo, si se considera que los bancos grandes tienen más del $60 \%$ de los activos del sistema bancario, son éstos lo que más provisionan por posibles pérdidas desde la cartera consumo (ver figura 11 del anexo digital).

En este sentido, el análisis del riesgo de crédito ex ante permite determinar que (1) la acumulación de provisiones totales no se ha detenido desde 2005, (2) el gasto de provisión ha alcanzado esto último, pero también a cubrir los créditos incobrables, y (3) el mayor gasto de provisión proviene de la cartera consumo, el mismo que se ha incrementado en el último lustro. De acuerdo a esto, hay que verificar, con base en el enfoque ex post, si en verdad la cartera consumo es la más riesgosa y si las provisiones acumuladas están justificadas.

Si bien es cierto que la literatura económica toma como variable los indicadores de cobertura con base en las provisiones por posibles pérdidas crediticias para verificar su incidencia en la rentabilidad de los bancos, Rodríguez (2015) afirmó que la relación no era significativa con base en una mejor definición de riesgo de crédito, la que se genera ex post y no ex ante: el nivel de morosidad del crédito. Este autor considera a esta última un resultado de factores macroeconómicos, políticos, institucionales y sociales, por un lado y, por otro, de la política bancaria de cada banco.

Al respecto, la morosidad de las carteras de créditos disminuyó lentamente en los primeros años de la dolarización, pero después de 2004 la caída fue más rápida hasta 2008, excepto la cartera consumo que se detuvo en el segundo semestre de 2006. En el primer semestre de 2009, todas las morosidades por tipo de cartera aumentaron, pero nunca regresaron a los niveles de los primeros años de la dolarización (ver figura 12 del anexo digital).

Cuando la economía se recuperó, en el último trimestre de 2009, de los estragos de la crisis financiera internacional de septiembre de 2008, la morosidad de los distintos tipos de cartera cayó lentamente y se evidenciaron dos resultados importantes: (1) la morosidad de la cartera consumo se mantuvo en niveles muy altos, por encima del promedio, y con un comportamiento al alza, y (2) la morosidad de la cartera comercial se ubicó por debajo de la registrada en las otras carteras de crédito —incluso de la cartera de vivienda desde enero de 2011-. Con la segunda restricción externa en el último trimestre de 2014, la morosidad promedio de la cartera comercial y vivienda aumentó levemente, no así de la cartera de consumo que creció bruscamente. 
Ecuador: la prudencia financiera de la banca privada en dolarización (200I-20I9) GI

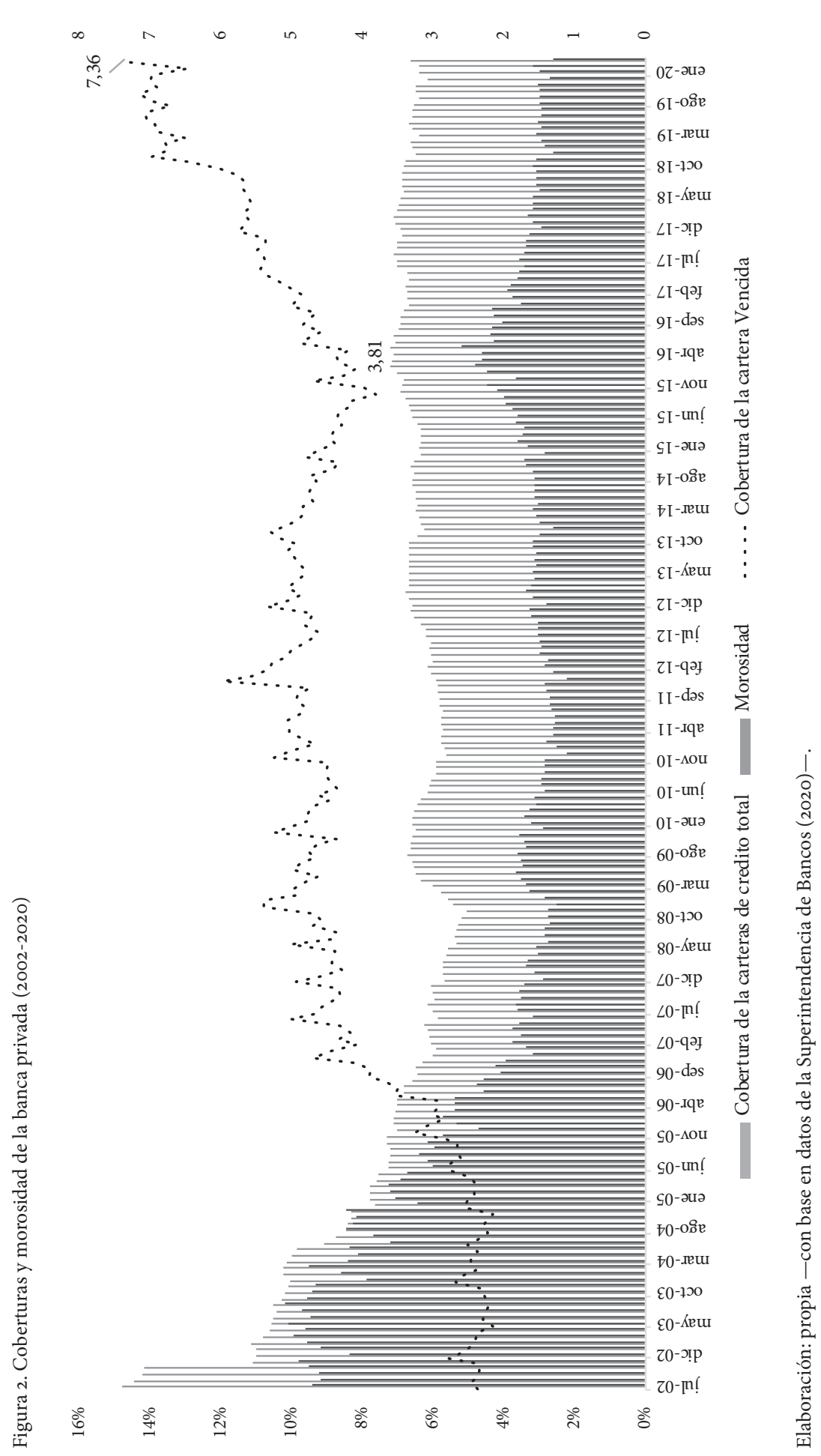


La cartera con mayor riesgo de crédito es la de consumo, mientras que la comercial es la de menor problema de incumplimiento y recuperación, de hecho, su morosidad ha disminuido en todo el periodo hasta ubicarse por debajo de todas las demás. Sin embargo, la banca privada orientó el crédito año a año en mayor proporción al consumo en desmedro de la cartera comercial, los dos segmentos con mayor peso en la cartera total. Por lo tanto, el crédito se orientó hacia la cartera de mayor riesgo y cada vez menos hacia la cartera de menor riesgo. A pesar de esto, el coeficiente cartera vencida/cartera total se ubicó en 0,94 \% en diciembre de 2019 (ver figura 13 del anexo digital).

El riesgo de crédito puede medirse según las decisiones de crédito pasadas (enfoque ex ante) o actuales (expost). El problema de realizar un estudio con el primer enfoque es que las instituciones financieras establecen niveles de provisión mínimos y ésta es una variable predeterminada. No obstante, se verifica que el sistema bancario ecuatoriano no opera con bajos niveles de provisión, todo lo contrario, mantiene altos coeficientes de cobertura: la provisión acumulada fue 7,01 veces superior a la cartera vencida y representó el 6,16 \% de la cartera total en diciembre de 2019 (ver Figura 2).

Si los indicadores de cobertura se los compara con los niveles de morosidad (riesgo de crédito ex post), resulta que la banca privada es altamente solvente y líquida. El indicador de cobertura de la cartera de crédito total puede cubrir una morosidad tan alta como la registrada en diciembre de 2004, cuando todavía le faltaba mucho por descender. Así lo demuestra la Superintendencia de Bancos (2019) cuando afirma que «en el escenario base estresado e hipotético, todos los tamaños, es decir, bancos grandes, medianos y pequeños, quedan por encima del nivel mínimo de solvencia del $9 \%$ [...] En las pruebas de tensión de liquidez se determina que todos los tamaños de bancos registran una resistencia de 3 meses» (pág. 11). A diciembre de 2019 , el nivel de solvencia se reduciría de $13,47 \%$ a $13 \%$ si es un «escenario estresado» y a 9,98\% si es hipotético, porcentajes por encima de lo que exige las normas de Basilea.

Si el mismo análisis se limita para la cartera consumo, la amplitud para enfrentar un posible aumento del riesgo de crédito se reduce. La cobertura de la cartera de crédito consumo total ha estado sobre la cartera vencida — resultado final de un crédito problemático-, pero no está por encima de la morosidad de esa cartera. Si el total de la cartera morosa terminara bajo la categoría de vencida, la provisión acumulada correspondiente no la podría cubrir. Todo esto sin tomar en cuenta los «otros rubros» de la provisión acumulada. Éste es el riesgo que corre la banca al maximizar su rentabilidad, el mismo que controla con base en el cortoplacismo o la alta preferencia por la liquidez de esta cartera (ver figura 14 del anexo digital).

El análisis comparativo no puede ser solo entre el riesgo de crédito probable (ex ante) y el real (ex post), sino también frente a países de América Latina. Según Felaban (2020), a junio de 2019, Ecuador fue el país con la más baja cartera vencida en la región, condición que se repite para los años anteriores, aunque compartiendo ese lugar con otros países como Nicaragua, Guatemala y Panamá.

Si el sistema bancario tiene coeficientes de cobertura superiores a la morosidad de las carteras de crédito y ostenta la cartera vencida más baja de América Latina, cabe indagar acerca de su nivel de provisión con respecto a los demás países. Si se considera el indicador de cobertura de la cartera vencida, el resultado no es causa de asombro: Ecuador fue el país, de lejos, con la 


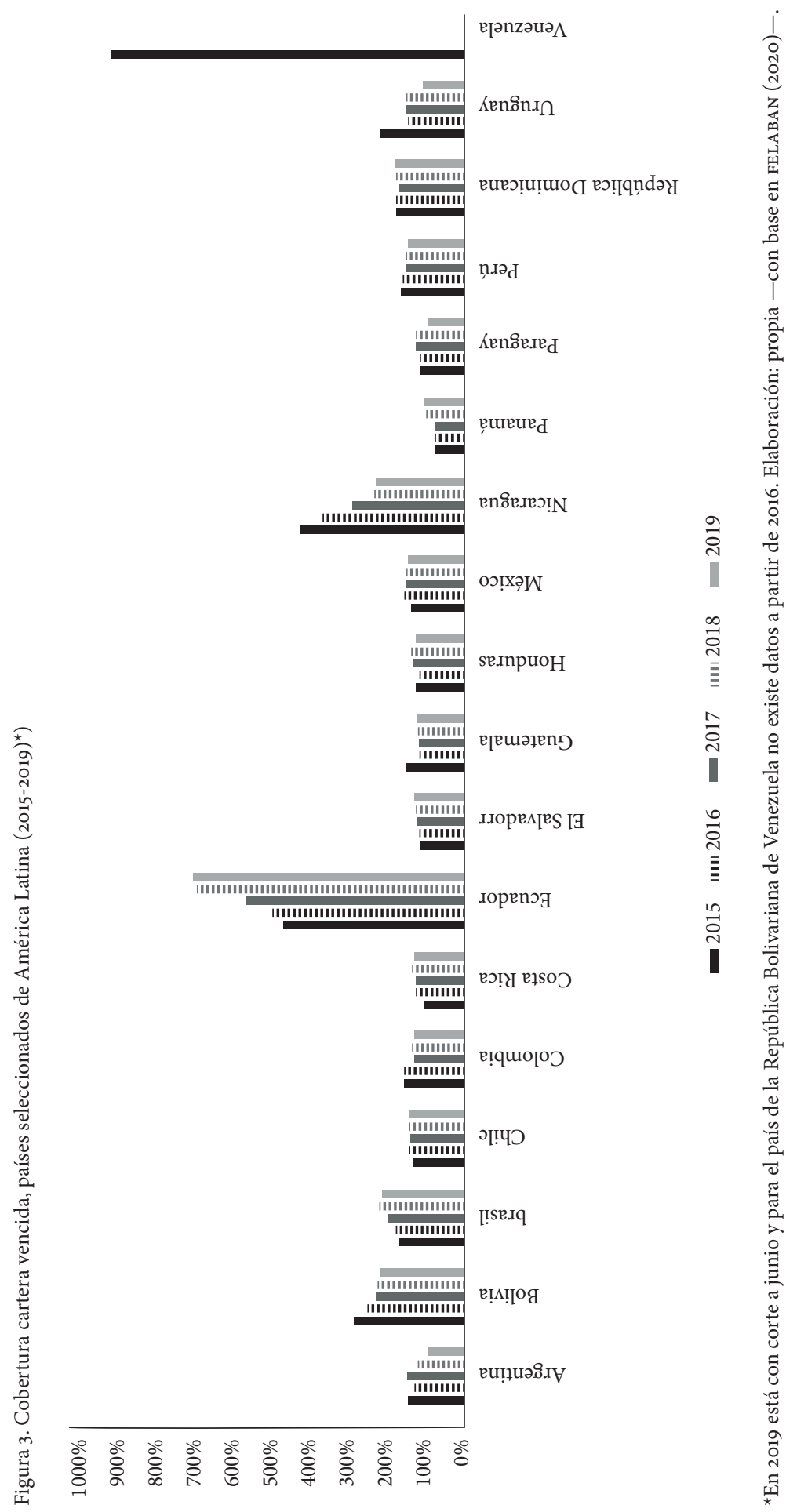


más alta cobertura en América Latina, solo por debajo de Venezuela. A junio de 2019, la provisión acumulada superó a la cartera vencida en 7,07 veces o representó 707 \% (ver Figura 3). ${ }^{6}$

Por estos indicadores, la banca privada ecuatoriana es considerada altamente conservadora. El gasto de provisión de las carteras de crédito contribuyó desde 2005 a la provisión acumulada total, sin excepción. Los años en que más aportó fueron 2011, 2012, 2014 y 2017, por encima del 50 \% (ver figura 15 del anexo digital). La contribución promedio del gasto de provisión entre 2005 y 2019 fue de 34,2\%.

Otro elemento: la Junta de Regulación Monetaria Financiera estableció el 12 de febrero de 2016 la constitución de provisiones para las entidades financieras públicas y privadas y detalló que para una cartera categoría A1 (crédito con probabilidad de cobro de $100 \%$ o fácil recuperación) debían generarse provisiones de hasta $1 \%$ (ver tabla 2 del anexo digital). Si se considera que el nivel de cartera total vencida generada por la banca a diciembre de 2019 fue 0,94 $\%$ y para la cartera de consumo vencida fue $1,26 \%$, la cobertura es muy alta para los dos casos. Como se observa en la figura 2 y en la 14 del anexo digital, la cobertura llegó para la primera a $6,16 \%$ y para la segunda a 3,33 \%. De esta manera, se constituyen provisiones no para una cartera de categoría A1 o A2, sino para una de más baja calidad, A3 y hasta B1.

\section{¿POR QUÉ TAN PRUDENTE Y CONSERVADORA?}

Las entidades financieras enfrentan diferentes tipos de riesgos, pero también la incertidumbre. Los riesgos se pueden medir, pero no la incertidumbre. No obstante, mientras mayores sean los riesgos, la economía está cada vez más expuesta a la crisis si se cumplen los eventos inciertos. A través del análisis realizado, se encontró que la banca privada es altamente conservadora y prudente frente al entorno económico. Por lo tanto, la primera causa de este tipo de comportamiento es enfrentar los diferentes tipos de riesgos y por los eventos inciertos.

Sin embargo, en este estudio se determinó que la liquidez es suficiente y las provisiones son extremadamente altas para enfrentar el aumento del riesgo de crédito, aún en los escenarios más complicados. Estos últimos, en la economía ecuatoriana, son sin duda los relacionados con la caída del precio del petróleo y/o los shocks externos, crisis económicas causadas por las restricciones vinculadas a la dinámica global como la de septiembre de 2008 y 2015 . La solvencia y los amplios recursos de la banca corrigieron el alza de la morosidad de las carteras y la falta de liquidez en esas dos crisis, incluidos eventos inciertos que se llegaron a cumplir como el terremoto de abril de 2016.

A pesar de la eficacia y el desempeño de los bancos para enfrentar la baja del ciclo económico en una economía como la de Ecuador, la alta preferencia por la liquidez evidenciada tanto en la orientación del crédito (cortoplacismo) como en los fondos disponibles y en el agresivo aumento de las provisiones por potenciales pérdidas, cuestionan el compromiso de las entidades financieras respecto a la movilización del ahorro y la mitigación de los problemas estructurales de la economía. Además, el alto gasto en provisiones puede afectar los ingresos del fisco y la política de fortalecimiento del patrimonio a través de una mayor reinversión de utilidades.

La segunda causa está vinculada directamente con la dolarización: la inexistencia de un Prestamista de Última Instancia (PUI). En una economía sin moneda propia, la banca debe 
formar su propio fondo de liquidez para hacer frente a una excesiva salida de depósitos y/o a la pésima colocación de los créditos. No obstante, desde 2008 fue creada la red de seguridad financiera, un sustituto cercano del PUI.

Por último, la banca privada ecuatoriana ha considerado imprescindible cambiar el marco regulador y adherirse a las normas de Basilea III. Éstas tienen como objetivo «crear bases para un sistema bancario resiliente que ayude a evitar la acumulación de vulnerabilidades sistémiCas» (ASOBANCA, 2019, pág. 18). Además de sugerir la mejora de la calidad del capital regulador bancario para absorber pérdidas, también añade «elementos macroprudenciales a la regulación» como los fondos de contingencias. Desde la visión de los banqueros ecuatorianos, éstos no solo deben estar formados por los bancos privados, sino también por el sector público. En el caso de Ecuador, la creación de fondos petroleros (ASOBANCA, 2018b).

\section{REFLEXIONES FINALES}

El comportamiento bancario en el Ecuador, en los años de la dolarización, se ha caracterizado por mantener altos niveles de liquidez y solvencia, muy por encima de las normas de Basilea III y del actual cuerpo legal y normativo. Junto con el cambio de la orientación crediticia para maximizar la rentabilidad, la banca privada ha demostrado ser bastante prudente y conservadora, lo que ha incluido el aumento drástico de las provisiones para créditos incobrables. Ante la inexistencia de un PUI, los representantes de la banca han solicitado que el encaje depositado en el Banco Central quede inmovilizado y que el Gobierno central, a través de la constricción de la política fiscal, lleve a cabo la creación de abultados fondos de contingencias.

Justifican estos reiterados pedidos no solo con base en las exigencias de una economía dolarizada y a los requerimientos de Basilea III, sino también en aplicar una política crediticia más laxa. Es razonable, pero en los 20 años de la dolarización, está comprobado que la banca privada ha racionado el crédito a los sectores que generan mayor valor agregado en sus procesos de producción y ha impulsado el consumo mucho más que la inversión, y las actividades económicas poco productivas, como la importación de bienes de consumo durable, especialmente vehículos. Asimismo, la banca, con su alta preferencia por la liquidez, en muchos años injustificada, ha obstaculizado un crecimiento económico alto y sostenido, ya que ha contribuido a la ineficiencia de la economía ecuatoriana.

\section{NOTAS}

1 Agradecemos la asesoría de Laura Vera, Carmen Lucero y un colaborador anónimo.

2 Para acceder al Anexo digital de este artículo, diríjase al siguiente enlace: https://uceedu-my.sharepoint. com/:b:/g/personal/revistaeconomia_uce_edu_ec/EaDpe_Tvsvxoru1GFEuBGykBo6nHmtcF8zovax3g$83 \mathrm{a} 5 \mathrm{~g}$ e $=$ fmeogp

3 En 2015, la Superintendencia de Bancos dispuso un nuevo segmento: la cartera de crédito productivo. Éste se desagregaría principalmente de la cartera de crédito comercial y es esencialmente de largo plazo. 4 Los indicadores de cobertura pueden ser dos: (1) provisión acumulada total/cartera vencida y (2) provisión acumulada total/carteras de crédito total.

5 Si se considera la provisión acumulada para carteras de crédito (no se incluyen los otros rubros), 2016 
fue el único año en que disminuyó desde 2005. Por lo tanto, la cartera vencida fue cubierta en su totalidad en 2016 por la suma del gasto de provisión y por el 16,92 \% de la provisión acumulada para carteras de crédito del año anterior.

6 Según Asobanca (2018a), la banca privada considera que el indicador de cobertura es la provisión acumulada sobre cartera improductiva - cartera vencida más cartera que no genera intereses- (pág. 5), cálculo que discrepa del expuesto por FELABAN (2020).

\section{REFERENCIAS}

Asociación de Bancos del Ecuador (ASOBANCA) (2018a). Cobertura: relación entre las provisiones y la cartera improductiva (84). Recuperado de https://www.asobanca.org.ec/sites/default/files/Revista\%20 Asobanca\%2oEd\%2084\%20CS6Baja.pdf

Asociación de Bancos del Ecuador (Asobanca) (2018b). Boletín macroeconómico. Recuperado de https://www.asobanca.org.ec/publicaciones/bolet\%C3\%ADn-macroecon\% $\mathrm{C}_{3} \% \mathrm{~B}_{3}$ mico/ bolet\% $\mathrm{C}_{3} \% \mathrm{ADn}$-macroecon\% $\mathrm{C}_{3} \% \mathrm{~B}_{3}$ mico-noviembre-2018

Asociación de Bancos del Ecuador (ASOBANCA) (2019). Informe técnico: estándares regulatorios financieros internacionales. Recuperado de https://www.asobanca.org.ec/publicaciones/estudios-especiales/ informe-t $\% \mathrm{C}_{3} \% \mathrm{~A} 9 \mathrm{cnnico}^{-e s t} \% \mathrm{C}_{3} \%$ Aindares-regulatorios-financieros

Cardim de Carvalho, F. J. (2015). Liquidity Preference and Monetary Economies. New York, usa: Routledge.

Chena, P., Bucella, M. y Bosnic, C. (2018). Efectos de la financierización en el cambio tecnológico en América Latina. En M. Abeles, E. Pérez, y S. Valdecantos (eds.), Estudios sobre financierización en América Latina (94-139). Santiago de Chile, Chile: Naciones Unidas/CEPAL.

De la Cruz y Alcántara (julio, 2011). Crecimiento económico y el crédito bancario: un análisis de causalidad para México. Revista de Economía, 28(77), 13-38.

Federación Latinoamericana de Bancos (FELABAN) (2020). Estadísticas indicadores homologados. Recuperado de https://indicadores.felaban.net/indicadores_homologados/

Fitzgerald, V. (2007). Desarrollo financiero y crecimiento económico: una visión crítica. Revista Principios, (7), 5-28.

Hermann, J. (2014). Restricciones financieras del desarrollo económico: teoría y políticas para los países en desarrollo. Revista CEPAL, (114), 71-87.

Hung, F. S. (2005). Credit rationing and capital accumulation with investment and consumption loans revisited. [Racionamiento de crédito y acumulación de capital con préstamos de inversión y consumo revisados]. Journal of Development Economics, 78(2), 322-347.

Junta de Regulación Monetaria Financiera (2016). Resolución $N^{\circ}$ 209-2016-F por la cual se norma la calificación de activos de riesgo y constitución de provisiones por parte de las entidades de los sectores financiero público y privado bajo el control de la Superintendencia de Bancos. Quito, Ecuador: Junta de Regulación Monetaria Financiera.

Keynes, J. M. ([1936]2007). Teoría general de la ocupación, el interés, y el dinero. Buenos Aires, Argentina: Fondo de Cultura Económica.

Keynes, J. M. ([1937]2017). Teorías alternativas de la tasa de interés. Revista de Economía Institucional, 19(36), 335-346.

King, R. G. and Levine, R. (1993a). Finance, entrepreneurship, and growth. Theory and evidence, Journal of Monetary Economics, (32), 513-542.

McMillan, J. (2018). El fin de la banca: el dinero, el crédito y la revolución digital. Madrid, España: Taurus. 
Paredes, G. J. (2015). Integraciones monetarias pasivas en Sudamérica (1991-2011). Buenos Aires, Argentina: Ediciones Cooperativas.

Paredes, G. J. (2017). Ecuador: ¿por qué salir de la dolarización? Revista CEPAL, (121), 149-167.

Paredes, G. J. y López, G. (noviembre de 2019). El racionamiento crediticio del sistema bancario ecuatoriano: una aproximación desde los empresarios del sector manufacturero. En M. Toscanini (Presidencia), Desafíos en el ámbito económico y empresarial: Cambios en el entorno en factores como la innovación, tecnología, mercados y competitividad. Ponencia presentada en el Primer Congreso Internacional en Ciencias Económicas y Empresariales, Guayaquil, Ecuador.

Petria, N., Capraru, B. and Ihnatov, I. (2015). Determinants of banks' profitability: Evidence from EU 27 banking systems. Procedia Economics and Finance, (20), 518-524.

Rodríguez, R. C. (2015). Morosidad en el pago de créditos y rentabilidad de la banca comercial en México. Revista Mexicana de Economía y Finanzas. Nueva Época/Mexican Journal of Economics and Finance, 10(1), 71-83.

Saona, P. (2016). Intra-and extra-bank determinants of Latin American Banks' profitability. International Review of Economics \& Finance, (45), 197-214.

Superintendencia de Bancos (2019). Reportes de pruebas de tensión. Recuperado de http://estadisticas.superbancos.gob.ec/portalestadistico/portalestudios/wp-content/uploads/sites/4/downloads/2020/02/pruebas_tension_dic_19.pdf

Superintendencia de Bancos (2020). Boletín de Series Bancos Privados. Recuperado de http://estadisticas.superbancos.gob.ec/portalestadistico/portalestudios/?page_id $=791$

Tapia, E. (13 de enero de 2020). La banca necesita mejores reglas. Revista Líderes. Recuperado de https:// www.revistalideres.ec/lideres/banca-reglas-creditos-indicadores-economia.html

Vera, C. y Titelman, D. (2013). El sistema financiero en América Latina y el Caribe Una caracterización. Santiago de Chile, Chile: CEPAL.

Vera-Gilces, P., Camino-Mogro, S., Ordeñana-Rodríguez, X. and Cornejo-Marcos, G. (2019). A look inside banking profitability: Evidence from a dollarized emerging country. The Quarterly Review of Economics and Finance. (s. d.).

Xu, M. T., Hu, K. and Das, M. U. S. (2019). Bank Profitability and Financial Stability. International Monetary Fund. (s. d.). 